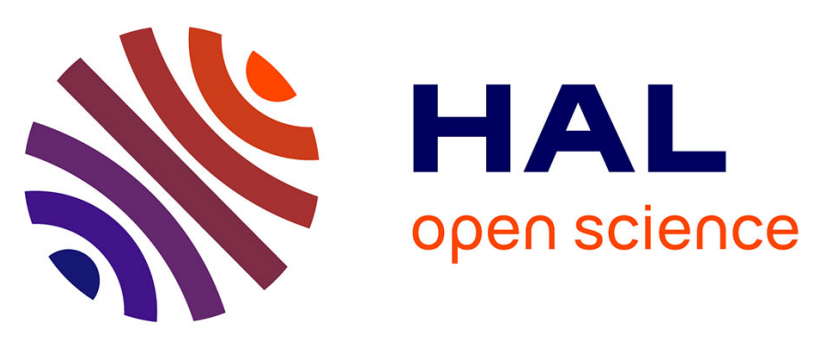

\title{
Experimental and Numerical Study of Internal Charging on Spacecraft and Risks of Discharge on Floating Metallic Elements
}

\author{
Abdessamad Ben Zaid, Thierry Paulmier, Pierre Sarrailh, Bernard Dirassen, \\ Romain Rey, Denis Payan
}

\section{To cite this version:}

Abdessamad Ben Zaid, Thierry Paulmier, Pierre Sarrailh, Bernard Dirassen, Romain Rey, et al.. Experimental and Numerical Study of Internal Charging on Spacecraft and Risks of Discharge on Floating Metallic Elements. IEEE Transactions on Nuclear Science, 2019, pp.1-9. 10.1109/TNS.2019.2958368 . hal-02422788

\section{HAL Id: hal-02422788 \\ https://hal.science/hal-02422788}

Submitted on 23 Dec 2019

HAL is a multi-disciplinary open access archive for the deposit and dissemination of scientific research documents, whether they are published or not. The documents may come from teaching and research institutions in France or abroad, or from public or private research centers.
L'archive ouverte pluridisciplinaire HAL, est destinée au dépôt et à la diffusion de documents scientifiques de niveau recherche, publiés ou non, émanant des établissements d'enseignement et de recherche français ou étrangers, des laboratoires publics ou privés. 


\title{
Experimental and Numerical Study of Internal Charging on Spacecraft and Risks of Discharge on Floating Metallic Elements
}

\author{
A. Ben Zaid, T. Paulmier, P. Sarrailh, B. Dirassen, R. Rey, and D. Payan
}

\begin{abstract}
Space system design rules and manufacturing processes may sometimes lead to the use of floating conductors in internal subsystems. This configuration promotes charge accumulation and increases the risk of electrostatic discharges. This paper presents an experimental and numerical study on this issue. We have selected and tested a Sub-D connector that is susceptible to internal charging because it may contain ungrounded conductors. We assessed its charging behavior and the risk of discharges induced by floating pins. For this purpose, the connector is tested in different configurations. It is irradiated using mono-energetic electron beams, carried out in the GEODUR irradiation facility installed at ONERA (Toulouse, France). Electric currents and potentials induced within the irradiated connector have been measured and correlated with simulation performed with SPIS-IC code. Experiments revealed that the connector's charging behavior is directly influenced by energy of the involved particles. The sample could present fast charging kinetics after exposure for a few hours at relatively low electron fluxes. Electrostatic discharges, intense enough to damage sensitive electronic components, have also been observed. We have shown that the discharge risk is mainly related to the connector wiring.
\end{abstract}

Index Terms-Charging kinetics, Electrostatic Discharges (ESD), Floating metal, Internal Charging, Spacecraft charging.

\section{INTRODUCTION}

$\mathrm{S}$ PACECRAFT around Earth are exposed to energetic particles from the radiations belts. Electrons and protons of high energy can be stopped or can go through the satellite's shielding, depending on their energy. The highest energy particles penetrate deep inside electronic boxes and are able to charge all the ungrounded internal materials such as: printed circuit boards (PCB) [1], cable dielectrics, connectors, integrated circuit packaging, capacitor boxes, etc. Mostly electrons participate to this phenomenon, because of their penetration range.

Along the time, the electrical charges accumulate in material, until the creation of a differential electric potential

A. Ben Zaid, T. Paulmier, P. Sarrailh, B. Dirassen, and R. Rey are with ONERA, The French Aerospace Laboratory, 31055 Toulouse, France (corresponding author e-mail : Abdessamad.Ben_Zaid@onera.fr).

D. Payan is with CNES, The French Space Agency, 31401 Toulouse, France. between the charged elements and the satellite's structure. This phenomenon is called Internal Charging. The electrical charge deposition creates potential differences that lead to intense electric fields in some cases. In addition, along the path of the high energy electrons, radiation dose is deposited in the material that can lead to the ionization of the material. As such, in dielectrics, the excited electrons and holes created by ionization increase the conductivity inside the material. If the conductivity is low enough, the rate of charge build-up can overcome leakage currents and the created electric field can exceed the breakdown threshold for the material [2].

Following the electric fields configuration, an ESD (Electrostatic Discharge) and/or electric arc can be initiated. A secondary effect of these phenomena is that it could produce electromagnetic disturbances that can interfere with satellite's on-board electronics, generating errors that could be compared to SETs (Single Event Transient) and could even damage the most sensitive electronic components. In the long term, the ESD could also result in the degradation of electrical properties of internal insulators and lead to premature ageing [3]-[4].

In order to have a better knowledge about the topic, the internal charging has been the subject of several studies, which assigned this phenomenon as probably responsible of a large part of anomalies observed on flight [5]-[6]. This was also confirmed by some onboard measurements such as the SCATHA and the CRRESS spacecraft that embedded an experimental monitor of internal discharge made by Frederickson [7].

Our study fits into this context. It focuses on a device that is particularly sensitive to internal charging. This device is a DSubminiature connector used in space industry. It has been selected because it is frequently used in spacecraft's payload. Considering the number of connection between sub systems, each satellite contains a significant amount of connectors which made them interesting candidates for this study. It presents indeed considerable potential risks because of its configuration:

1) In most cases the wiring setup in satellites can not prevent some connector's contacts to be floating. It increases the risks of breakdowns and disruptive discharge.

2) This type of connector often makes connection between power sources (e.g. solar panels) and other devices. Hence, high currents are likely to pass through them, which can cause sustained discharges. 
3) The thickness of its dielectric is as large as $1 \mathrm{~cm}$, which is quite thick and thus favorable to charges accumulation.

The sample tested is showed in fig. 1. (a) It consists of 25 copper alloy pins, a steel shell or metallic holder (yellow chromate over cadmium/zinc) and a thermoplastic dielectric.

The main objective of this paper is the assessment of internal charge and discharge risk relying on experimental tests and numerical analysis. To achieve this objective the connector is tested in various setups which represents realistic inflight configurations. Then, the kinetics of charge and the occurrence of discharges on floating metal parts are analyzed. In this paper only the results of two setups are presented in details. The paper is divided into several parts:

In section II, the experimental setup and numerical simulation protocol are detailed. A special attention was payed to the measurement of ESD. In Section III, the first setup results are presented and analyzed before being compared with the simulation results in section IV. The last section is dedicated to the study of discharges produced under irradiation.

\section{EXPERIMENT SETUP AND SIMULATION PROTOCOL}

The test specifications must correspond to the internal charging conditions, i.e. using an electron source that allows irradiating with monoenergetic beams or with energy spectrum from $100 \mathrm{keV}$ to $2.5 \mathrm{MeV}$, with fluxes ranging from $10^{-1}$ pA.cm ${ }^{-2}$ to 1 pA.cm ${ }^{-2}$ corresponding to the threshold level defined using data from [8].

Our work has not been carried out under representative conditions of a specific real case, but under realistic conditions (in terms of flux and energy) which allow a better characterization of the charging kinetics and to charge enough to have high potentials able to trigger discharges. The results of the two test campaigns presented here are carried out using two monoenergetic electron beams, respectively at $400 \mathrm{keV}$ and $1.3 \mathrm{MeV}$ with fluxes ranging from $1 \mathrm{pA} . \mathrm{cm}^{-2}$ to $10 \mathrm{pA} . \mathrm{cm}^{-2}$. Each irradiation phase is followed by a relaxation time, during which the sample is not subjected to any radiative stress and we control the potential decrease over the time. For this purpose, ONERA's GEODUR facility has been used.

\section{A. GEODUR Facility [9]}

GEODUR is a radiation test facility allowing the study of satellite internal and surface charging, evaluation of RIC (Radiation Induced Conductivity) of thick materials and sample radiative ageing using $400 \mathrm{keV}$ to $2.5 \mathrm{MeV}$ monoenergetic electrons. It is equipped with a $2.5 \mathrm{MeV}$ Van de Graff electron accelerator and a double scattering system for the production of a distributed electron spectrum in the energy range [200 keV - $1 \mathrm{MeV}$ ]. It is instrumented with a contactless electrostatic probe and current measurement systems for the characterization of internal charging behavior of space elements. The temperature of the sample holder can be controlled in the range $\left[-180^{\circ} \mathrm{C},+250^{\circ} \mathrm{C}\right]$ allowing to reproduce the temperature variations on flight. A pumping system allows experiments at vacuum of around $10^{-6} \mathrm{hPa}$. This facility has been used in this study for irradiation at high energies and low electron flux. All tests were performed at room temperature in vacuum conditions about $10^{-6}$ mbar.

\section{B. Tests Setup}

Several setups have been tested during this study. Here, we itemize only "Setup 1". In "Setup 1", the sample's pins are wired as shown in Fig.1. (a). Among the 25 pins, nine pins are electrically floating (not connected to the ground), with two pins connected to metal disc labeled "flag 1" and two pins connected to "flag 2" (flags function is given below). The remaining pins are grounded and monitored through a picoammeter and current probes.

Most studies in scientific literature are measuring only the currents or the potentials [10]-[11]-[12]. In our work, we propose a setup to measure both simultaneously:

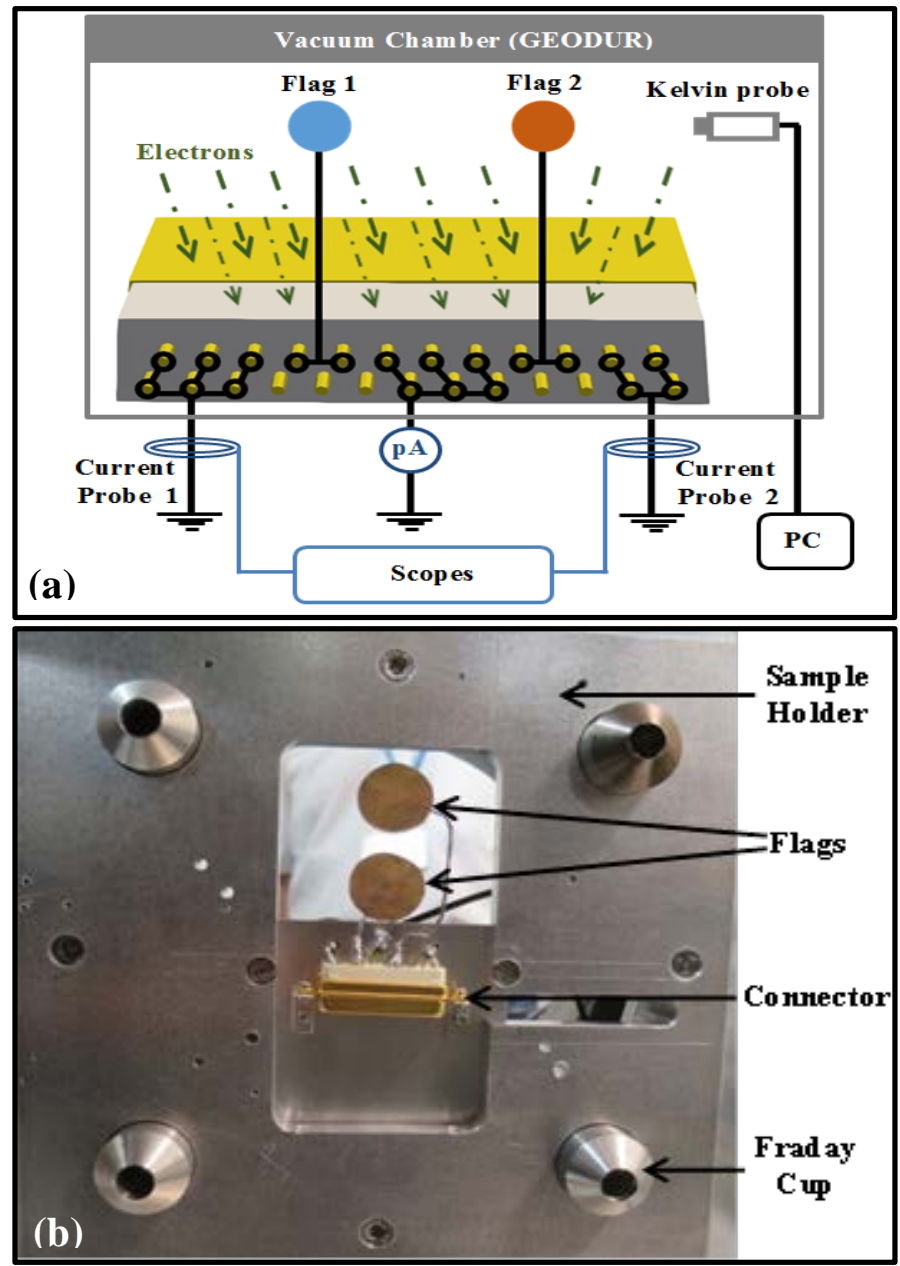

Fig. 1. (a): Test setup schematic, connector wired according to setup 1. (b): View of the DUT mounted on a metal sample holder in the Geodur facility. The current measurement instruments are positioned behind the sample holder.

The combination of these measurements allows firstly, detecting and characterizing the discharge using the measured current, secondly, knowing the triggering threshold of the discharge (potential measurement). These data correlated with simulation will give more information about the ESD, such as the electric field that could be compared with the material dielectric strength, the amount of charges evacuated, and the discharge energy. The surface potential is measured using a Kelvin probe which is placed in front of the surface to be measured. The minimum area needed to measure the potential is about $1 \mathrm{~cm}^{2}$. 
This is why two thin metallic disks called "flags" have been added to measure the ungrounded pins potential. These flags are circular, to avoid the introduction of edge effects that could modify the electric fields. Their area is small enough to reduce stray capacitance which is created in the vacuum by the distance between the flags and the sample holder.

The ESD is a very fast phenomenon. Its characteristic time scale is about from nanoseconds to microseconds. Due to ESDs, a transient current is then measured for a few nanoseconds. To detect and measure it, Pearson probes were used because they are sufficiently sensitive and offer a fairly fast response. They are connected to oscilloscopes that will get triggered and record the transients when the ESD occurs.

The measurement system was designed in order to avoid ESD signal disturbance due to the electromagnetic coupling induced by the electronics around and especially by the cable routing between the Pearson probes and the oscilloscopes.

Therefore, the electrical ground must be common to GEODUR and oscilloscopes, all instruments must be grounded, the wire through which the discharge current flows and the used cables must be very short without loops.

Without these protective measures, the discharge signals would be disturbed by system's parasitic inductances, capacitances, and impedances. Therefore, the signal would correspond more to typical RLC circuit response as shown by some results obtained by T.A. Schneider et al. [13].

If the dielectric becomes conductive due to a discharge, a leakage current could be present due to the creation of a conductive path between the dielectrics and the ground. This current is less important than the discharge current. To measure it, a pico-ammeter was used.

Finally, the DUT (device under tests) is installed on the GEODUR sample holder in such a way that only the sample is irradiated, while the flags are protected behind a shield. This shielding has been build up with metallic plate thick enough (about $3 \mathrm{~mm}$ ) to stop electrons of $1.3 \mathrm{MeV}$. This process aims at measuring floating pins potential only induced by the charges implanted in the material.

\section{Numerical Simulation Protocol}

The numerical study was divided into two distinct parts: Radiation and Charging. It requires the use of different tools forming a simulation chain, respectively Geant4/GRAS [14] and SPIS-IC. First, we create two connector models where materials properties are defined. The first one is based on GDML format [15] used by Geant4/GRAS. The second one is a GMSH format [16] used by the scoring algorithm of Geant4/GRAS and by SPIS-IC for the input data concerning the charge and dose deposition rates. In a first time, these two geometrical models are used to carry out the radiation simulation using Geant4/GRAS. We configure the irradiation: source, type of particles, energy, and number of events (based on the Monte-Carlo method). The output results are the charge deposit, the dose deposit, and the deposited energy normalized by the number of events.

In a second time, the charging simulation is carried out with
SPIS-IC (Spacecraft Plasma Interaction Software - Internal Charging). This simulation first requires the GRAS results. SPIS-IC evaluates charge transport, conduction levels, and potential or electric field induced by the irradiation. It is based on the resolution in 3D of three equations [17]:

1) The Poisson equation: relation between the net charge inside the computational volume and the electrical potential.

2) The continuity equation of the net charge: equation of evolution of the net charge as a function of the net current and the source term of the charge.

3) The Ohm's law: net current of the charge carrier as a function of the material conductivity and the electric field.

\section{TEST RESUlTS - CHARGING BEHAVIOR}

In this section, the experimental results are presented. Fig. 2 shows the surface potential evolution measured at the dielectric surface of the sample and on ungrounded pins connected to the flags. The first two steps were performed at $400 \mathrm{keV}$ at different flux values. It allows the observation of the charging dynamic's dependence on the flux value. Indeed, different surface potential kinetics are observed depending on flux value. It should be noted that these temporal variation of surface potential are quite linear, especially at the beginning of the irradiation. It means that the connector has a capacitive behavior. The conduction current is negligible with respect to the capacitive current. It is only valid at the beginning of the irradiation: over the time the potential increase becomes slightly less linear meaning that the conductivity increases with irradiation (the RIC effect). It becomes more visible after several days of irradiation: the insulator resistivity gradually decreases (as shown by the results with another setup presented in section V). However, during the first hours of irradiation the dielectric intrinsic properties are not yet affected, so we can calculate its charging kinetics: e.g. with a flux of $1 \mathrm{pA} . \mathrm{cm}^{-2}$ it is charged with a kinetics of $-4.8 \mathrm{~V} \cdot \mathrm{min}^{-1}$. A priori it presents no significant risks when the connector is used in this configuration (setup1) and under these conditions.

\section{A. The Charging Kinetics}

Comparing the irradiation phase at $400 \mathrm{keV}$ with the irradiation at $1.3 \mathrm{MeV}$ (after $\mathrm{t}=2700 \mathrm{~min}$ ), we can say that the charging kinetics is energy dependent. At $400 \mathrm{keV}$ the charging kinetics on the dielectric surface is higher than the one on the flags (floating pins), and the opposite at $1.3 \mathrm{MeV}$. This phenomenon could be explained by two physical processes governing the charge:

This difference would be related to the electron range into the dielectric. Using Weber's equation [18], we obtain a range of $0.6 \mathrm{~mm}$ at $400 \mathrm{keV}$ versus $3 \mathrm{~mm}$ at $1.3 \mathrm{MeV}$. Knowing that the flags are placed on pins located at $3 \mathrm{~mm}$ from the sample surface, the charges do not reach the pins at $400 \mathrm{keV}$ and the potential of the floating pins is steered by the amount of charge implanted in the dielectric and the associated capacitances. A second parameter that contributes to this process is the following:

The $400 \mathrm{keV}$ beam deposits charges near the surface (without 


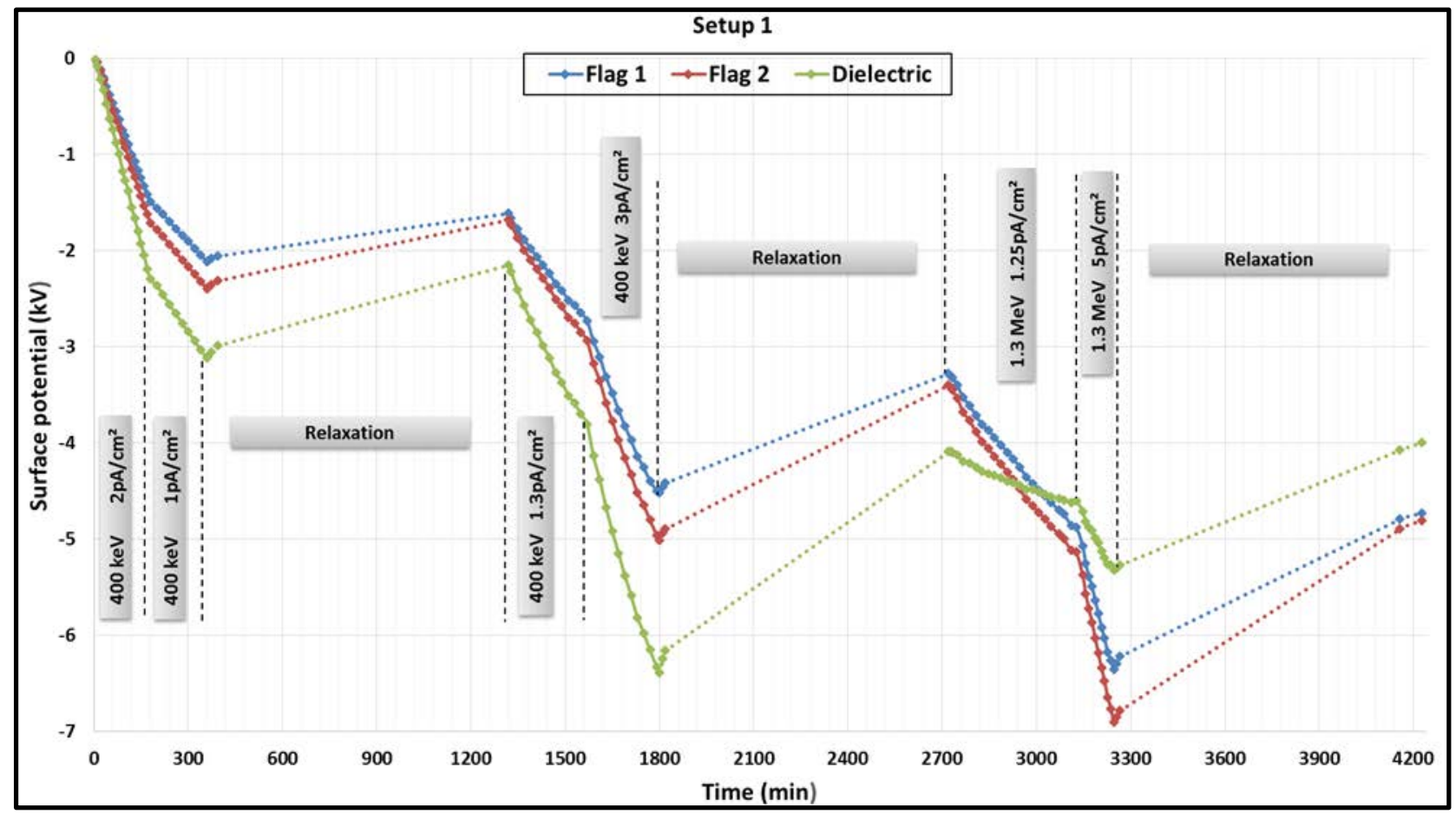

Fig. 2: The surface potentials measured during the test performed in Setup 1 . The solid lines represent periods during which the measurements are performed. In relaxation phase, the measurements are taken only in the beginning and at the end; it is represented here by dashed lines.

generating the RIC in the overall bulk), which induces higher potential there.

On the opposite, the $1.3 \mathrm{MeV}$ beam will induce significant radiation conductivity in the material. It leads to a transport of the charges deposited on the surface and drifting then towards the dielectric's volume where the pins connected to the flags are located. As a result, the flags potential increases faster than the dielectric's surface potential. This is confirmed by the numerical analysis in Section IV.

2) This difference would also be steered by a non-uniform distribution of the electric field in the material, due to difference on the distances between pins/surface and the ground point.

\section{B. The Relaxation Kinetics}

After the irradiation at $400 \mathrm{keV}$, the pins' relaxation kinetics is lower than the dielectric's surface, and the opposite at $1.3 \mathrm{MeV}$. It means that the deep implantation of charges (at 1.3 $\mathrm{MeV}$ ) facilitates their flow to the ground via the grounded pins and the RIC generation enhances this flow. There is also an electric field effect on RIC: relaxation is high in the area where the field is more intense, which is in accordance with Poole-Frenkel law [19].

The last point that emerges from the curves in fig. 2 is the difference between the flags. Flag 1 charging kinetics is slightly lower than flag 2 one. This can be explained by the difference between internal electric field and internal capacitance configurations because the number of floating pins below the flags varies from one flag to the other (see Fig. 6 and explanation in section IV). In addition, the flags location is not the same (see Fig. 1) so the capacitive coupling between each flag and the sample holder is different.

Other setups have been tested, for which pins wiring is done in such a way as to balance the internal electric field, i.e. pins connected to flags are at the same distance from ground and from other ungrounded pins. The results confirm what has been described earlier.

\section{NUMERICAL ANALYSIS}

In this section, the simulation results are presented, in order to complete and validate the explanations given in section III.

\section{A. Results Comparative}

Fig.3 shows the simulation results compared to the surface potential measurement during the $400 \mathrm{keV}$ irradiation phase (Fig. 2 limited to $2100 \mathrm{~min}$ ). Overall, it can be seen that a good qualitative agreement is obtained between the simulations and the experimental results regarding the charging kinetics, especially during the first irradiation phase (2 and $1 \mathrm{pA} / \mathrm{cm}^{2}$ ). From a quantitative point of view, the discrepancies can be attributed to the uncertainties on different parameters:

1) The capacitive coupling of flag with the surrounding metallic elements (sample holder, connector shell, wire connecting flag to pins, second flag). Due to a complex configuration, the capacitive coupling is not accurately estimated. It also affects the others pins capacitance and therefore the dielectric.

2) An underestimated conductivity (more details later) as shown in the second irradiation phase, the charge evolution gradually loses its linearity while during simulation it remains linear.

3) Measurement process: The Kelvin probe averages the potential over a $1 \mathrm{~cm}^{2}$ areas only on the dielectric surface (it does not cover the entire surface). In simulation, we have plotted the maximum potential measured on the dielectric, which does not necessarily correspond to that of its surface. Indeed, the potential distribution is not uniform in the dielectric. 


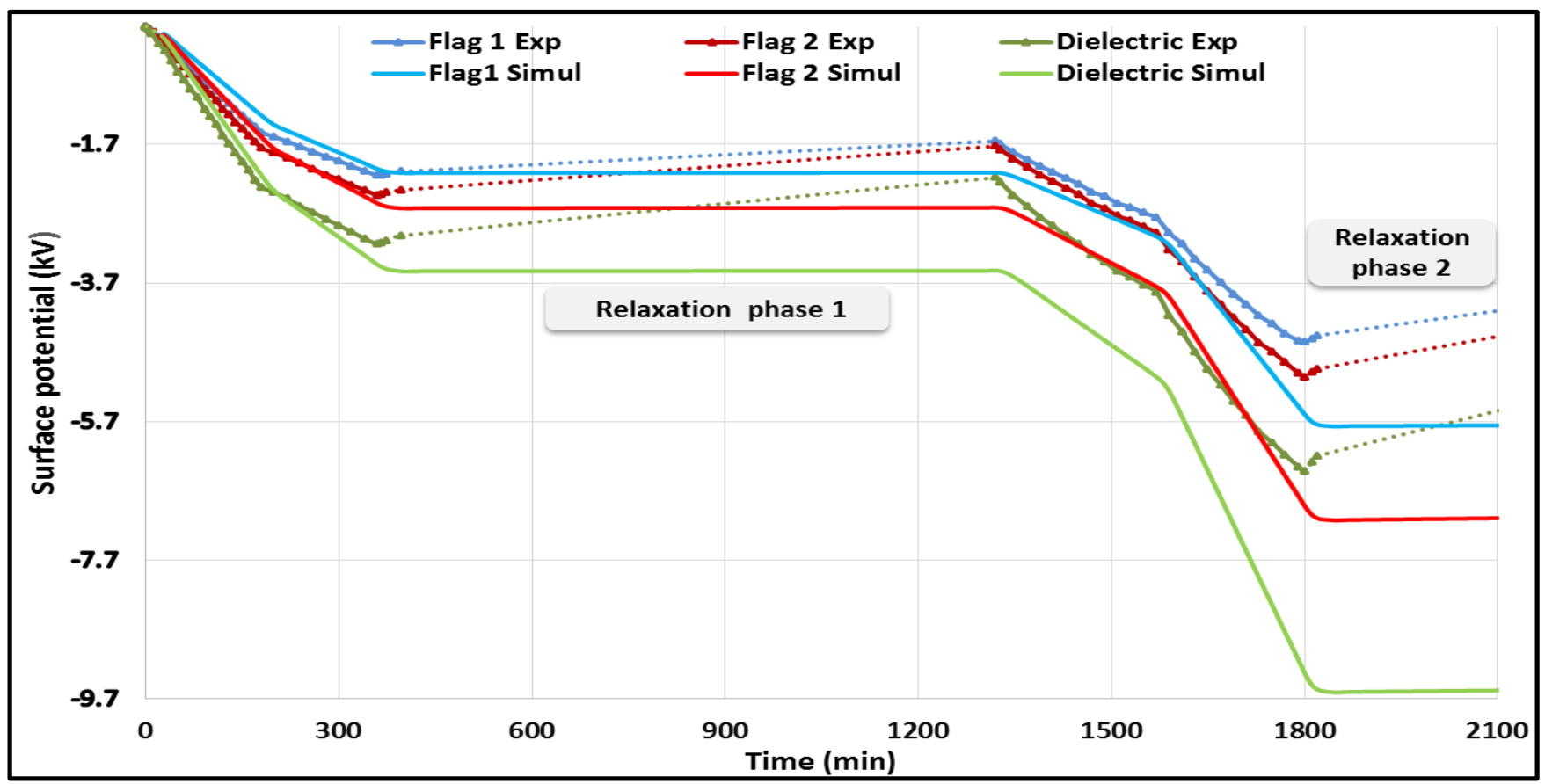

Fig. 3: Comparative surface potentials obtained during the $400 \mathrm{keV}$ irradiation phase. Simulation performed with same fluxes than experiment

Focusing on relaxation kinetics, fig. 3 shows that the kinetics is higher in the second phase compared to the first phase: this is valid for both results. For numerical relaxation this effect is visible when extending the curves up to 2700min (due to lack of space and for a better readability of the curve, we have cut it at 2100min whereas it normally lasts 2700min.). This highlights the production and increase of RIC over time. However, it is important to note that numerical relaxation is very low compared to the experimental one (contributing to the increase of the observed discrepancy between charging levels after the first relaxation phase.). We attribute this lack of relaxation to the underestimation of conductivity during relaxation. This is due to the uncertainty of material's electrical properties defined for radiation, which are not always easy to extract accurately, because for the same material, they may vary from one sample to another depending on the manufacturing process (e.g. density). This directly impacts the dose deposition map used to compute conductivity with SPIS-IC. There is also another phenomenon not taken into account in the code: it is related to physics at the materials interface (shell/dielectric): in fact, the transition between theses materials affects the particle transport, which introduces uncertainty about the charge and the dose deposition.

In addition to all these uncertainties, the conductivity model itself could be involved in the discrepancies noted between results (see the conclusions of P. Sarrailh et al. [17]).

\section{B. Energy Effect on Charging Behavior ${ }^{\ddagger}$}

As described in section III, the connector parts charge differently depending on the irradiation energy. This effect is illustrated through the maps of various parameters presented in the following figures.

In Fig. 4, we can therefore see the effect of the electron range:

\# Sometimes the notation "1MeV" is used to indicate "1.3MeV" at $1.3 \mathrm{MeV}$ charges are deposited from the dielectric surface to the first pins row (where the flags are connected), they are

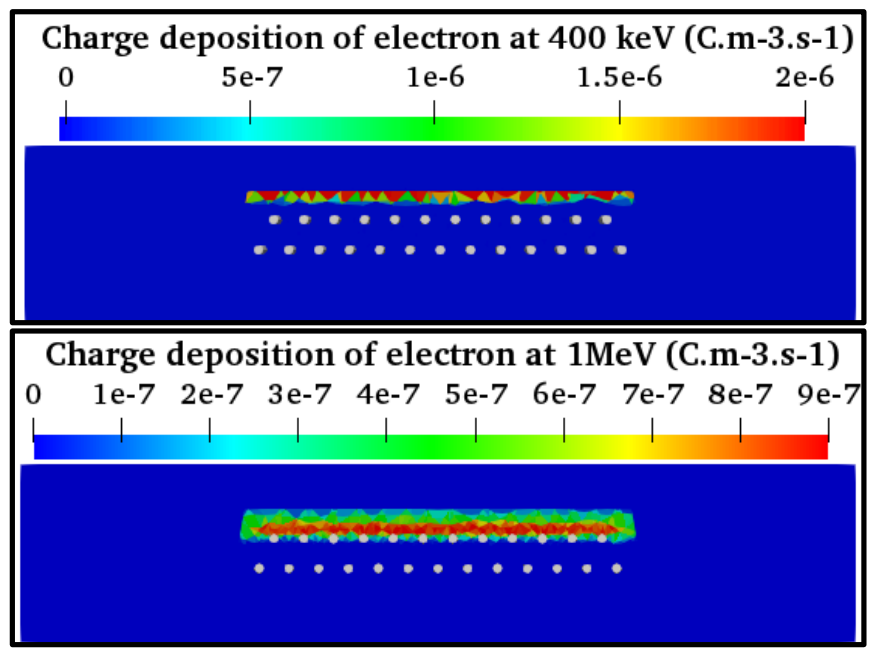

Fig. 4: Charge deposition rate at $400 \mathrm{keV}$ and at $1.3 \mathrm{MeV}$

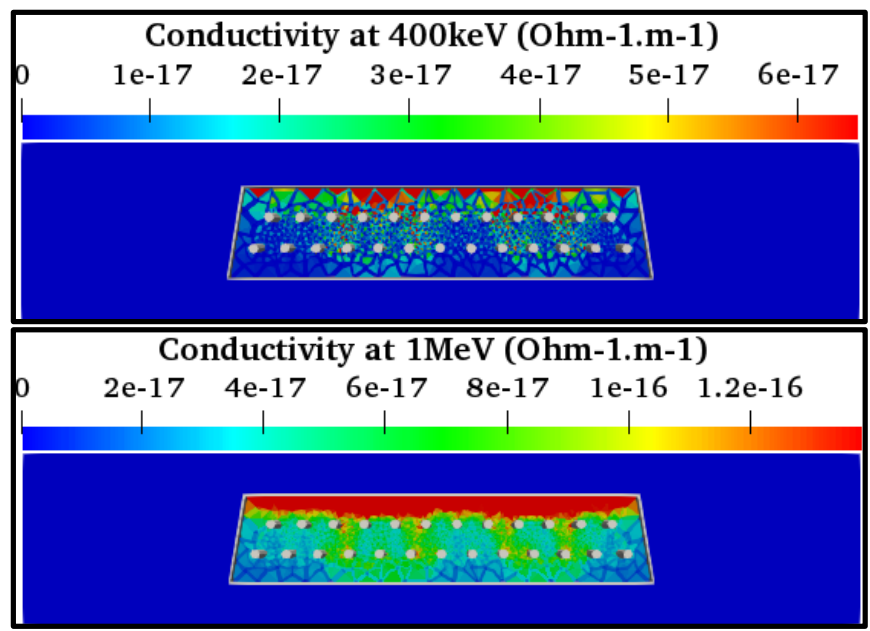

Fig. 5: Electrical conductivity map at $400 \mathrm{keV}$ and at $1.3 \mathrm{MeV}$ 
focused right above the pins, in contrast to $400 \mathrm{keV}$ for which the charges are limited to the connector surface resulting in a high potential on the surface. Both profiles are picked up where the dielectric is directly exposed to radiation, eliminating the effect of the shielding (connector shell).

In Fig. 5, the electrical conductivity maps are presented. Overall, conductivity is higher at $1.3 \mathrm{MeV}$ than at $400 \mathrm{keV}$. In addition, at $1.3 \mathrm{MeV}$, it is quite uniformly distributed in the volume, with a maximum between the sample surface and the area where the charges are deposited. This means that the current is strongly ionizing in this case. On the opposite, the current at $400 \mathrm{keV}$ rather contributes to charges accumulation on the dielectric surface. In both cases we observe conductivity concentration (with more or less high levels) around the floating pins. This is due to the electric field effect the distribution profiles of which are being provided in fig 6 .

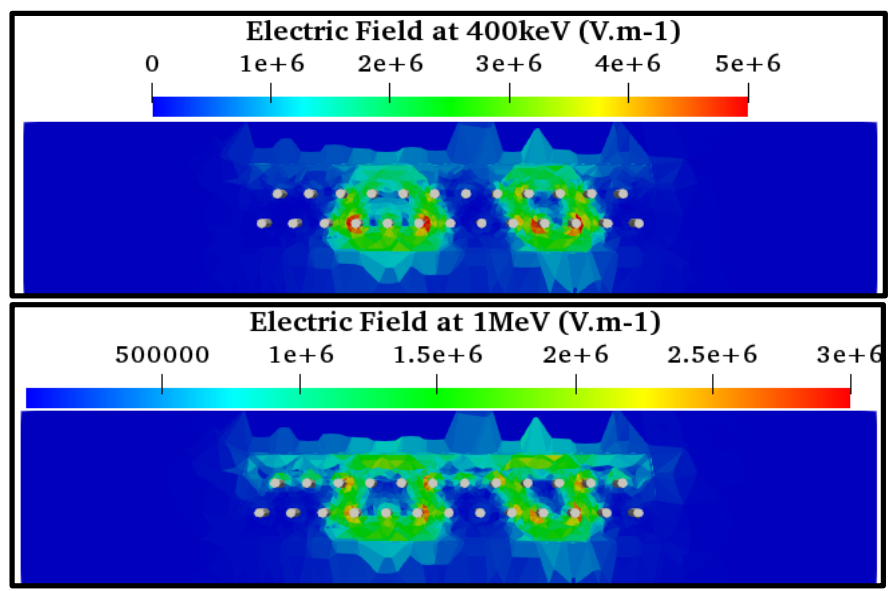

Fig. 6: Electric field maps at $400 \mathrm{keV}$ and at $1.3 \mathrm{MeV}$

Overall, the highest levels of the electric field were measured with the $400 \mathrm{keV}$ irradiation. Through the observation of the maps presented in Fig.6, showing that the field is not homogeneous in the dielectric volume, we can state that the electric field is steered by two mechanisms:

1) In proximity to electric ground: the field is high in the area between the grounded pins and the floating pins, more precisely on the side facing the grounded pin (red spots). It is in these areas that the current is strongly conducted (through the dielectric to ground). The distance is an important factor because although the connector shell is grounded, the field remains smaller between the ungrounded pins and the shell because the distance between them is larger than the distance between the pins themselves. It is therefore more probable that discharge will occur between a floating pin and an adjacent grounded pin. This applies to all floating pins except the one below flag 1 (the fifth from the left of the bottom row), which is close to the bottom part of the shell: the yellow spot underneath this pin, indicating a fairly high electric field, can be interpreted as an orientation of the conducted current that seems to flow towards the shell.

2) The second effect is that of capacitance effect of the floating pins: the field is strong where the capacitance is low. This is why the field of pins connected to the flags is smaller than the completely floating pins.

Through these comparisons between $400 \mathrm{keV}$ and $1.3 \mathrm{MeV}$ we observed that: at low energy the material is highly charged close to the surface, bulk conductivity is low and the electric field can reach significant levels. At high energy, electron irradiation deeply implants charges and generates ionization which cans results in some conductivity level that favors the charges evacuation and homogenization in the material: therefore potential differences are limited and electric fields are decreased. We can deduce that low energies could present a larger risk of charging and discharging than high energies. Following this reasoning, this would be applicable to energy spectra representative of realistic environments. The risk level would therefore depend on the spectrum shape. A spectrum where high energy electrons are predominant should minimize the risk of discharges, and vice versa. This raises an obvious question: at what value can energy be considered high enough to be "safe"? This requires further investigation and certainly depends on several parameters including the type and layout of the target device.

\section{Discharges on Sub-D ConNeCtor FloAting PinS}

This section is dedicated to discharges analysis. In contrast to Setup 1, other configurations have resulted in several ESDs. In this paper only an example of discharge current is provided, it has been measured during the second test campaign when the connector is wired according to setup 4 showed Fig. 7. (b).

\section{A. Discharge Characterization}

In order to allow a better ESD characterization, the potentials evolution during this test is given in Fig 7. (a). It should be noted that the potential curves are not linear compared to the

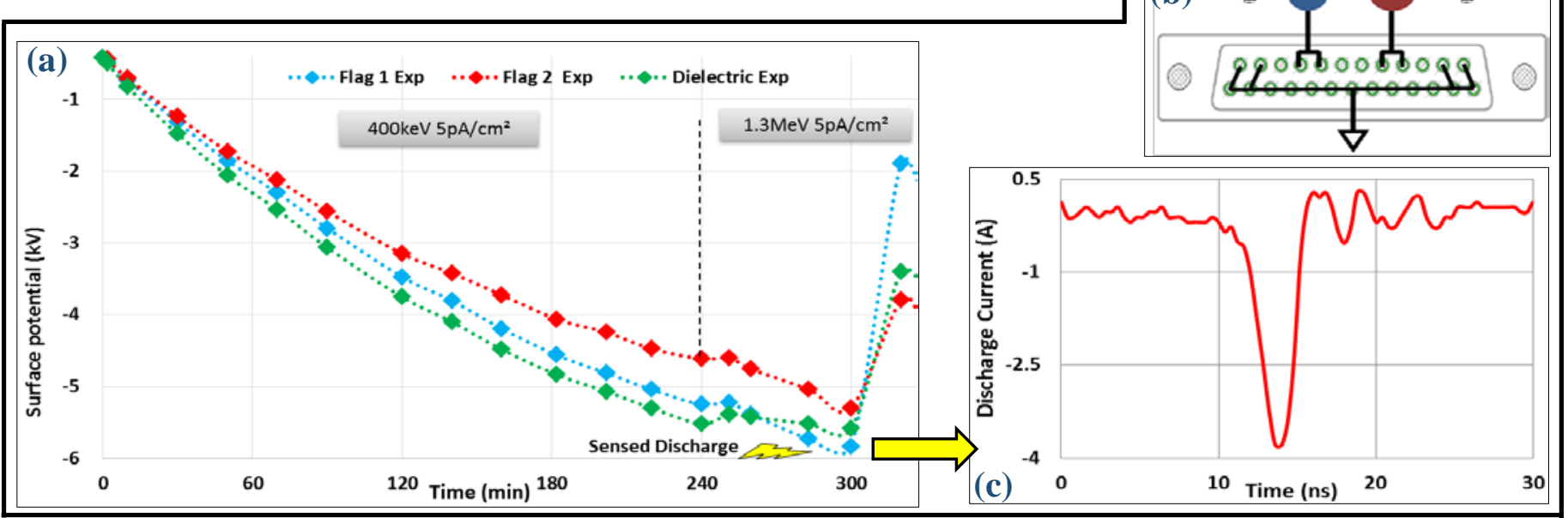

Fig. 7. (a): The surface potentials measured during the test performed in Setup 4.

(b): Connector wiring schematic during the setup 4.

(c): The transient current waveform recorded during the discharge 
first test (setup 1, Fig. 2), this is mainly attributed to a progressive ionization (generated during the earlier tests: setup1, setup 2 and setup 3) which increases the conductivity inside the dielectric. It is also probably due to a degradation of the material's electrical properties. This degradation is manifested through the decrease of the discharge triggering potential level in this case it is equal to $-5.7 \mathrm{kV}$, whereas it is between $-7.5 \mathrm{kV}$ and $-8.5 \mathrm{kV}$ for the previous tests (setups 2 and setup 3).

The final important point revealed by this graph is the location where the discharge occurs: it seems that it occurred around or near the pins connected to the flag 1; in fact, as shown Fig. 7. (a), flag 1 has the largest decline of potential: $4.3 \mathrm{kV}$ versus $2.1 \mathrm{kV}$ and $3.2 \mathrm{kV}$ respectively to flag 2 and the dielectric surface.

The electric field map measured (not represented here), during simulation, at 300min (the time when the discharge occurred) confirms this and reveals that the maximum field is around the floating pin located on the left of flag 1 . With a strong potential and surrounded by several grounded pins, it has therefore initiated the discharge. The maximum field measured (simulation) is about $3.5 \times 10^{6} \mathrm{~V} \cdot \mathrm{m}^{-1}$. This seems small compared to the dielectric strength of the material $\left(1.4 \times 10^{7} \mathrm{~V} . \mathrm{m}^{-1}\right)$. This information does not call into doubt the accuracy of the measured value because the dielectric strength provided for most materials is not an exact data. In other words, this data depends on the material thickness and cannot be considered in the case of a 3D material with a particular geometry such as that of the studied connector.

Fig. 7. (c). shows the waveform of the transient current measured during the discharge which occurred after 300min under irradiation. A peak current of about 3.8A is observed and the discharge duration is about 7 nanoseconds. From these data the total charge discharged by the ESD is estimated at $18 \mathrm{nC}$, the charge evacuated around flag 2 is estimated at approximately 9nC. This amount does not reflect the total charge contained in the dielectric. The simulation indicates indeed that the dielectric contains a total charge of $45 \mathrm{nC}$ at this moment. For this reason the potentials of Fig.7. (a) are not at zero right after the discharge.

This discharge would be much more intensive and would generate a much higher current with a higher energy if the conductivity was lower.

However, the measured current remains relatively high compared, for example, to the input currents allowed by most integrated circuits. It is therefore probable that this discharge could damage the adjacent electronic circuits by propagation, and generate considerable electromagnetic interferences.

\section{B. Discharge risks depending on setups}

A total of five setups have been tested during this study, in order to determine in which setup the connector is more at risks:

Discharges have been observed in all setups except setup 1, on the other hand, setup 2 is the most favorable to discharges occurrence (8 ESDs), followed by the 5 (7 ESDs). From this observation, a comparative study between the various setups has been established using setup 1 a reference. A simulation of the three setups was therefore carried out under the same conditions and with identical parameters: duration, flux and energy (400 keV). Then the electric fields were extracted at a key moment in the simulation, when the potential differences are the maximum. Thereby, the internal distributions of electric fields are presented in Fig. 9.

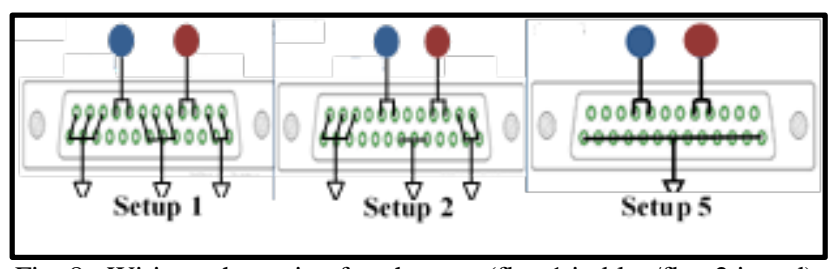

Fig. 8: Wiring schematic of each setup (flag 1 in blue/flag 2 in red)
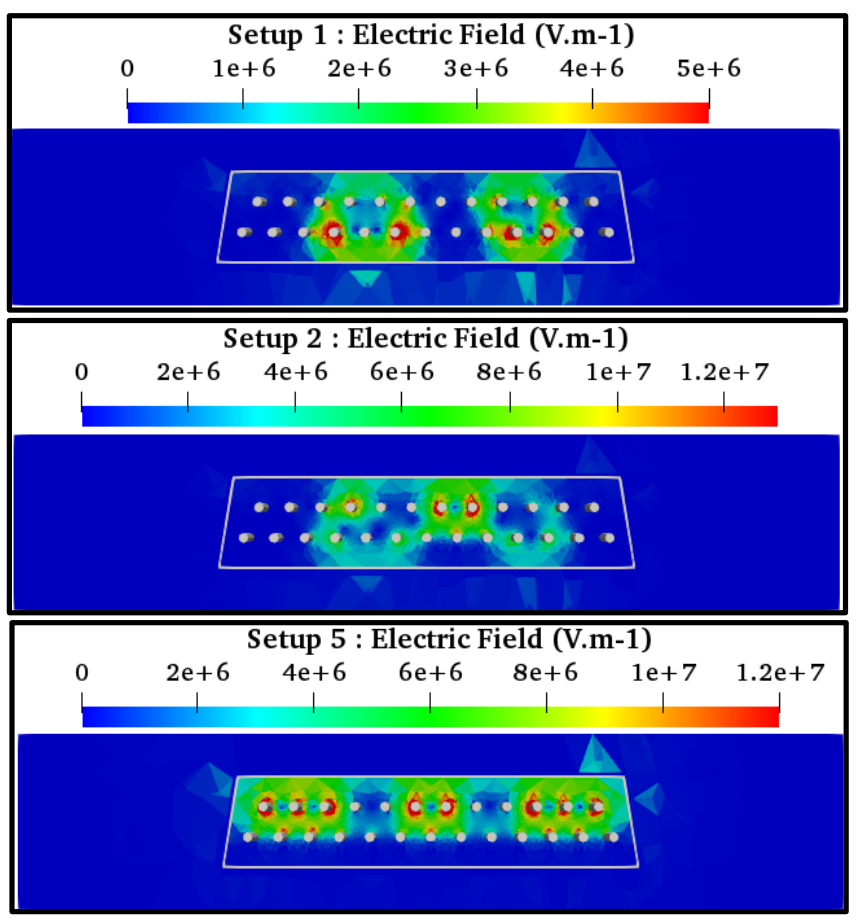

Fig. 9: Electric field maps of each setups

At first sight, we can notice that among the three configurations, setup 2 has the highest field level $\left(>1.2 \times 10^{7} \mathrm{~V} \cdot \mathrm{m}^{-1}\right)$, setup 5 is positioned in the second position with maximum field strength equal to $1.2 \times 10^{7} \mathrm{~V} \cdot \mathrm{m}^{-1}$ and lastly setup 1 with $5 \times 10^{6} \mathrm{~V} \cdot \mathrm{m}^{-1}$. This confirms the experimental results. Therefore, we can affirm that the essential criteria determining the configurations sensitivity are the number and location of floating pins:

- Setup 1 is the least critical because the number of its ungrounded pins is lower than that of the other ones.

- Setup 5 is less hazardous than setup 2 because the floating pins of setup 5 are arranged in a homogeneous way that gives a certain balance to the electric field (which is relatively uniform).

Another factor involved in raising the risk of discharges is the vicinity of the ungrounded pins to the area where the charges are deposited, thus generating considerable potential. This concerns the pins of the top row, as illustrated by the field profile of setup 5, and also visible on setup 2 (the two pins between the flags and the pin to the left of flag 1). 
As explained in the previous section, the capacitance of floating conductors also has a significant role in the electric field distribution, which is why the field is less important on ungrounded pins that are connected to the flags. Hence, for a device with floating conductors, it would be strongly advised to connect the floating parts together in order to increase their capacitance and thus minimize the breakdowns risk.

\section{CONCLUSION}

This paper presents a part of the study carried out on a Sub-D connector, which has been characterized with regard to the internal charging phenomenon. Overall, the results show that the sample tends to charge quickly and strongly due to the very high dielectric thicknesses which therefore involve very low dielectric capacitances. Regarding discharges, the sample presents significant risks of triggering harmful ESDs generating EMCs, particularly because of the pins shape which generates intense electric fields. Leaving some pins at floating potential increases this effect. To minimize this effect it is suggested to move the floating pins away from ground points and the area that may contain charges, it is valid not only for the connector but also for other devices with unconnected conductors.

It would seem that exposure to environments with a high concentration of low-energy electrons may increase breakdown risks in internal charging, especially when the target system has floating metal elements placed near the surface. To reduce this risk, shielding of inner materials and components (to eliminate the lower part of the spectrum) could be a solution. But how will this shielding affect the spectrum part with high energies electrons? This could reduce the radiation induced conductivity and, therefore, the ability to flow the charges from the sample to ground. Moreover, this effect certainly depends on material properties such as its conduction and its behaviour towards high-energy electrons. So, further studies are needed to assess the effect of internal shielding on charging behavior.

There are very few criteria allowing the characterization and/or assessment of breakdown risks with a high degree of accuracy. Often, the information available in the literature is the dielectric strength of materials, but this study has shown that this is not a reliable parameter. Indeed, in the case of systems with complex geometries, the risk levels cannot be estimated on the basis of this parameter.

The last step of this study will be to test a system consisting of cable and connector. A similar study is underway on floating PCB conductors.

\section{ACKNOWLEDGMENT}

The authors would like to thank CNES, the French Space Agency, for financial, technical and scientific support. They would also like to thank Artenum Company for the provision of the Edge software (ExtendeD Gdml Editor).

\section{REFERENCES}

[1] E. P. Wenaas, M. J. Treadaway, T. M. Flanagan, C. E. Mallon, and R. Denson, "High-Energy Electron-Induced Discharges in Printed Circuit Boards,” IEEE Trans. Nucl. Sci., vol. 26, no. 6, pp. 5152-5155, Dec. 1979.

[2] J. Sorensen et al., "ESA's tools for internal charging," IEEE Trans. Nucl. Sci., vol. 47, no. 3, pp. 491-497, Jun. 2000.

[3] K. Yagai, H. Miyake, Y. Tanaka, and T. Takada, "Internal charging phenomena and change of electrical properties in electron beam irradiated insulating materials for spacecraft," in 2011 Annual Report Conference on Electrical Insulation and Dielectric Phenomena, Cancun, Mexico, 2011, pp. 125-128.

[4] T. Paulmier et al., "Aging Effect and Induced Electric Phenomena on Dielectric Materials Irradiated With High Energy Electrons,” IEEE Trans. Plasma Sci., vol. 41, no. 12, pp. 3422-3428, Dec. 2013.

[5] H. C. Koons et al., "The Impact of the Space Environment on Space Systems," presented at the 6th Spacecraft Charging Technology Conf, 2000, pp. 7-11.

[6] G. L. Wrenn, "Conclusive evidence for internal dielectric charging anomalies on geosynchronous communications spacecraft,” J. Spacecr. Rockets, vol. 32, no. 3, pp. 514-520, May 1995.

[7] A. R. Frederickson, "Electric Discharge Pulses in Irradiated Solid Dielectrics in Space,” IEEE Trans. Electr. Insul., vol. EI18, no. 3, pp. 337-349, Jun. 1983.

[8] M. D. Violet and A. R. Frederickson, "Spacecraft anomalies on the CRRES satellite correlated with the environment and insulator samples,” IEEE Trans. Nucl. Sci., vol. 40, no. 6, pp. 1512-1520, Dec. 1993.

[9] T. Paulmier, B. Dirassen, M. Belhaj, V. Inguimbert, D. Payan, and N. Balcon, "Experimental test facilities for representative characterization of space used materials," presented at the Spacecraft Charging Technology Conference (13th SCTC), Pasadena US, 2014.

[10] J. J. Likar, K. Hartojo, G. Ott, J. Bird, and N. P. Meredith, "Spacecraft Charging Related Risk of Floating Connector Pins," IEEE Trans. Plasma Sci., vol. 46, no. 1, pp. 201-206, Jan. 2018.

[11] J. Chinn, W. Kim, N. Low, E. Martin, D. Thorbourn, and M. Smithfield, "Internal Electrostatic Discharge Tests of Micro-D Connectors for Planned Europa Mission,” p. 27, 2017.

[12] W. Kim, I. Katz, N. W. Green, and I. Jun, "An internal electrostatic charging test of circuit boards under electron beam," in 2011 12th European Conference on Radiation and Its Effects on Components and Systems, Sevilla, Spain, 2011, pp. 767-770.

[13] T. A. Schneider et al., "Deep Charging Evaluation of Satellite Power and Communication System Components," presented at the Spacecraft Charging Technology Conference (14th SCTC), ESA/ESTEC Noordwijk, 2016, p. 17.

[14] G. Santin, V. Ivanchenko, H. Evans, P. Nieminen, and E. Daly, "GRAS: a general-purpose 3-D Modular Simulation tool for space environment effects analysis," IEEE Trans. Nucl. Sci., vol. 52, no. 6, pp. 2294-2299, Dec. 2005.

[15] "GDML." [Online]. Available: http://gdml.web.cern.ch/GDML/.

[16] "Gmsh: a three-dimensional finite element mesh generator with built-in pre- and post-processing facilities.” [Online]. Available: http://gmsh.info/. [Accessed: 31-Jan-2019].

[17] P. Sarrailh et al., "Validation Through Experiments of a 3-D Time-Dependent Model of Internal Charging," IEEE Trans. Plasma Sci., vol. 45, no. 9, pp. 2566-2572, Sep. 2017.

[18] K.-H. Weber, "Eine einfache reichweite-energie-beziehung für elektronen im energiebereich von $3 \mathrm{keV}$ bis $3 \mathrm{MeV}$," Nucl. Instrum. Methods, vol. 25, pp. 261-264, Dec. 1963.

[19] J. Frenkel, "On Pre-Breakdown Phenomena in Insulators and Electronic Semi-Conductors,” Phys. Rev., vol. 54, no. 8, pp. 647-648, Oct. 1938. 